\title{
Super Composite: Carbon Fibre Infused 3D Printed Tectonics
}

\author{
H. Mohamed ${ }^{1}$, D. W. Bao ${ }^{2}$, and R. Snooks ${ }^{1(\bowtie)}$ \\ 1 Royal Melbourne Institute of Technology (RMIT), Building 100, Melbourne 3000, Australia \\ \{hesam.mohamed, roland.snooks\}@rmit.edu.au \\ 2 Centre for Innovative Structures and Materials, School of Engineering, RMT University, \\ Melbourne 3001, Australia \\ nic.bao@rmit.edu.au
}

\begin{abstract}
This research posits an innovative process of embedding carbon fibre as the primary structure within large-scale polymer 3D printed intricate architectural forms. The design and technical implications of this research are explored and demonstrated through two proto-architectural projects, Cloud Affects and Unclear Cloud, developed by the RMIT Architecture Snooks Research Lab. These projects are designed through a tectonic approach that we describe as a super composite - an approach that creates a compression of tectonics through algorithmic selforganisation and advanced manufacturing. Framed within a critical view of the lineage of polymer 3D printing and high tech fibres in the field of architectural design, the research outlines the limitations of existing robotic processes employed in contemporary carbon fibre fabrication. In response, the paper proposes an approach we describe as Infused Fibre Reinforced Plastic (IFRP) as a novel fabrication method for intricate geometries. This method involves $3 \mathrm{D}$ printing of sacrificial formwork conduits within the skin of complex architectural forms that are infused with continuous carbon fibre structural elements. Through detailed observation and critical review of Cloud Affects and Unclear Cloud (Fig. 2), the paper assesses innovations and challenges of this research in areas including printing, detailing, structural analysis and FEA modelling. The paper notes how these techniques have been refined through the iterative design of the two projects, including the development of fibre distribution mapping to optimise the structural performance.
\end{abstract}

Keywords: 3D printing $\cdot$ Fibre composite $\cdot$ Additive manufacturing

\section{Introduction}

The focus of this paper is the application and development of a series of technical innovations that enable the design and realisation of complex, intricate architectural forms through a carbon fibre reinforced 3D printed polymer fabrication approach. This Infused Fibre Reinforced Plastic (IFRP) method offers a viable approach to constructing super composites that have intrinsically complex geometries and compressed tectonics. This strategy is part of a larger trajectory of design research undertaken by the Snooks Research Lab that explores the compression of the tectonic relationship between skin, structure, services, and ornament, through the composite fabrication of intricate algorithmic architecture (Snooks 2020) (Fig. 1). 


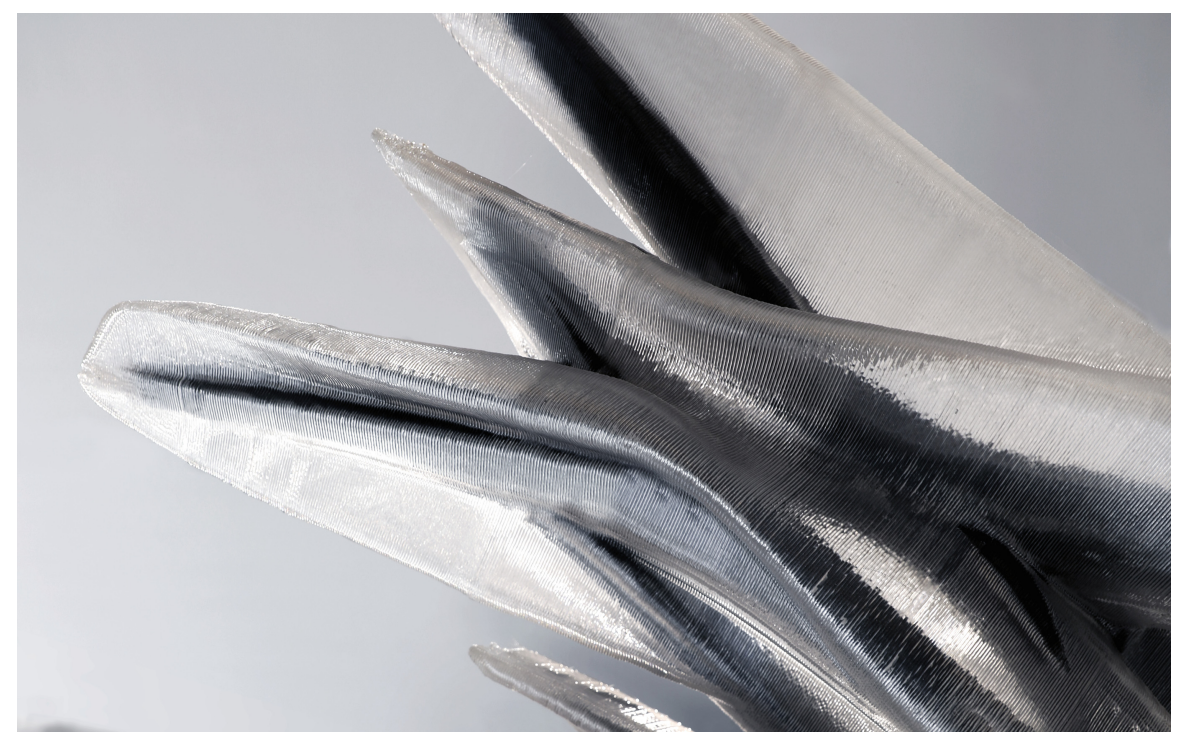

Fig. 1. Cloud Affects, 2019, detail photographs, Snooks Research Lab.

This research contributes to a growing international body of work and community of architects who are exploring the application of 3D printing in architecture. The research presented here has evolved from an exploration of the design implications of 3D printed translucent plastics and how these can be reinforced to enable their robust application to architecture. While Fused Deposition Modeling (FDM) is a relatively mature 3D printing technology, its application to large-scale architectural fabrication requires numerous innovations in terms of hardware, printing techniques, software development, architectural detailing, structural design, and the design of tectonics. This paper outlines some of these developments including printing topologically complex forms, the subdivision of form into printable parts, non-parallel printing techniques, the infusion of carbon fibre within printed conduits, structural analysis and design of carbon-infused plastic parts and jointing systems.

\section{Context}

\subsection{Fibre Composites in Architecture}

Advancements in compounding and fabrication techniques have paved the way for synthetic polymer composites to be deployed as a new building material. While the use of fibre composites in architecture dates to at least 1956 with the Monsanto House, recently there has been a re-emergence of polymer composites in architectural construction and discourse. This is evident in the work of architects such as Greg Lynn; with a long track record of innovative applications of composites through which he has realised complex curvature architectural forms. He describes "intricacy" as a tectonic relationship between elements with the continuous and gradient change to produce a dynamic spatial effect 
(Lynn and Friedman 2003). Lynn envisages a shift in architectural fabrication in which fusion by heat and chemical attachment replaces assemblage through mechanical connections (Lynn 2011a, b). Use of fibre reinforced polymer composites in Lynn's work allows for the gradual transformation of geometry to expose structure and alignment of load paths with the flow of surface.

\subsection{Robotic Fabrication and High-Tech Fibre Structures}

The repetition in production of fibre composite parts laminated onto traditional moulds create considerable efficiencies in sectors such as the marine industry to invest in reusable moulds. However, the architectural application of this approach is problematic when there is little repetition as the cost of the mould becomes prohibitive. Over the past decade architectural research groups including the Institute of Computational Design and Construction (ICD) in Stuttgart, and the Digital Building Technologies (DBT) lab at the ETH in Zurich have developed new robotic techniques for the fabrication of fibre reinforced polymers that avoid the use of moulds. These techniques, and the projects they are applied to, are important precursors to the research posited here. However, they embed specific limitations on architectural form. The ICD Stuttgart fibre winding projects, including the Research Pavilion (2010) and Buga Fibre Pavilion (2019) use customised jigs to create ruled surface geometry. An alternative approach explored by the ICD is robotic taping/gluing of a fibre structure to a temporary inflatable formwork (Doerstelmann et al. 2014). The DBT lab at ETH has experimented with a two-step fibre reinforcement of 3D printed form, where a composite of polymer and fibre is extruded directly on to a 3D printed base geometry (Kwon et al. 2019). Although this CFRP technique advances design research into fibre reinforced polymer structures, this method limits the topological scale and intricacy of the architectural forms that can be fabricated.

\subsection{Big Area Additive Manufacturing}

This paper is focused on Fused Deposition Modelling (FDM) of polymers within the field of additive manufacturing, also known as 3D printing. Big Area Additive Manufacturing or (BAAM) refers to the fabrication of large-scale components through FDM techniques. In the past few years, BAAM has become a desirable technique for relatively fast and efficient fabrication of components in different industries such as aerospace, automotive, product design and more recently within the fields of architectural design and building construction. 


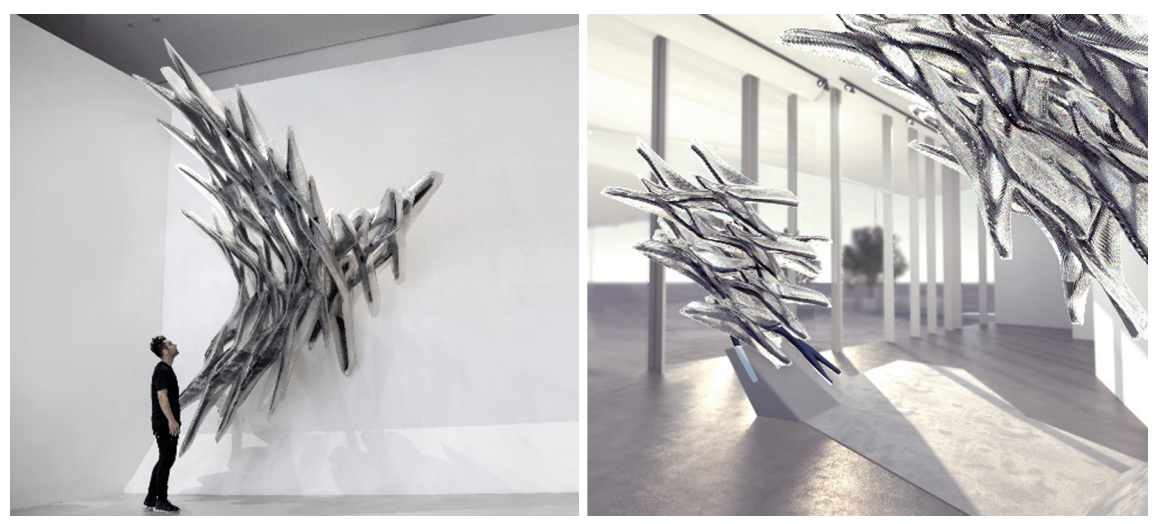

Fig. 2. Cloud Affects, 2019 (Left), and Unclear Cloud, 2020 (Right), Snooks Research Lab.

\section{Methodology: Intricate Tectonics and Composite Fabrication}

\subsection{Super Composite}

This research extends the concept of composites from material (fibre composite) to tectonics by compressing surface, structure, services and ornament into a single irreducible assemblage - a super composite (Fig. 3). This strategy is leveraged to develop intricate tectonics for expressive architectural forms. The super composite approach is designed through an agentBody self-organising generative algorithmic process that draws on the logic of swarm intelligence. It operates through multi-agent systems (agentBody algorithms form part of the Behavioral Formation design methodology developed by Roland Snooks since 2002). The agentBody generative design process simultaneously creates both the structure and form, as the agentBody consists of a skin and embedded structural skeleton (Snooks et al. 2020). The fabrication logic of the super composite leverages the geometric capacity of 3D polymer printing and the structural capacity of carbon fibre.

\subsection{A Lineage of 3D Printed Sacrificial Formwork Projects}

A super composite is a 3D printed hybrid of skin and embedded structural conduits that are infused or cast with structural material. The precursor to this approach was developed through a series of projects beginning with the NGV Pavilion (2016) Hawthorn Public Art (2016) by Studio Roland Snooks. These projects developed a tectonic, based on the logic of 3D printed sacrificial moulds, or formwork, into which high strength concrete structural elements were cast (Fig. 4) (Snooks 2018). This research has more recently been expanded to explore the implications of infusing carbon fibre into 3D printed sacrificial formwork conduits through the Cloud Affects and Unclear Cloud projects.

The infusion of carbon fibre within 3D printed conduits represents a novel approach to fibre composite fabrication that departs from the contemporary carbon fibre fabrication approaches, such as fibre winding and taping described above. Instead, this research employs a fibre infused sacrificial formwork technique to create a negotiated relationship between the inner and outer skins where form and structure negotiate one another to enable intricate topologically complex forms. 


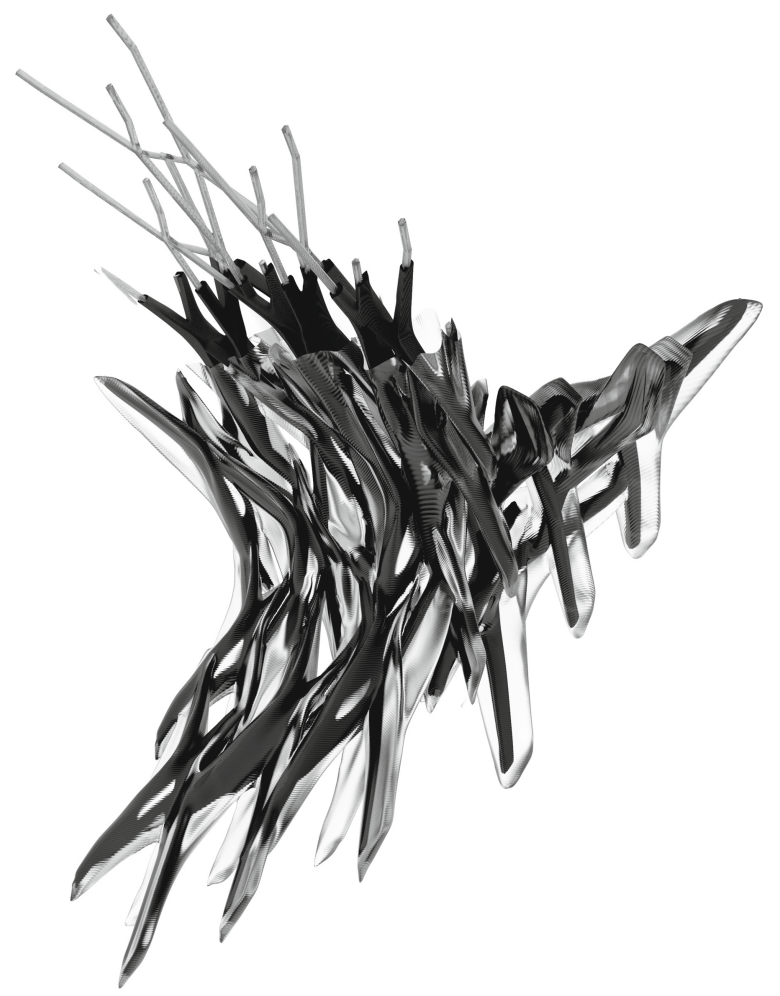

Fig. 3. Super composite: compressed structure, services, and skin
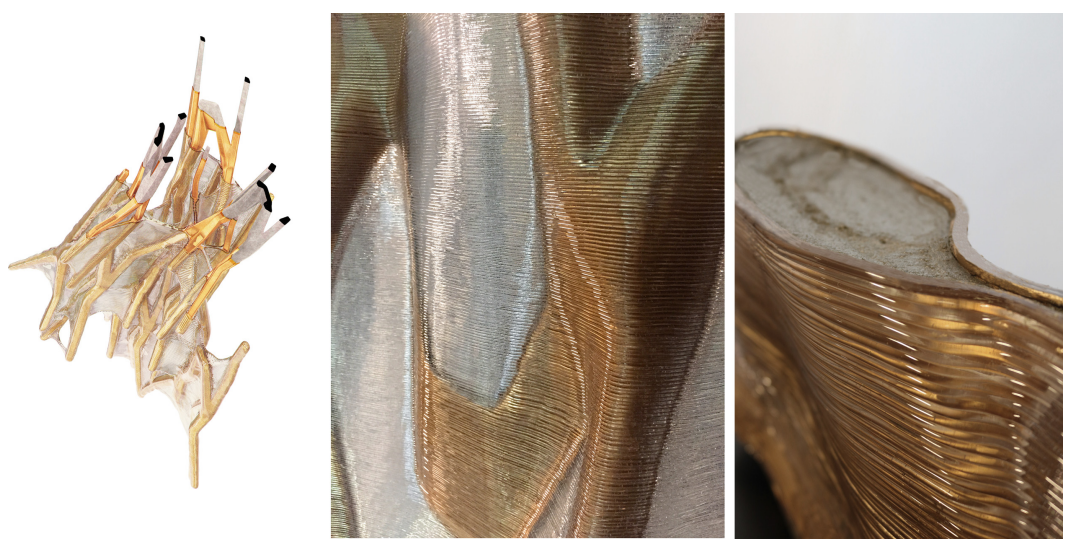

Fig. 4. Concrete infused sacrificial formwork, NGV Pavilion, 2016, Studio Roland Snooks 


\subsection{Sacrificial Formwork for Carbon Fibre Infusion}

In this approach, a multilayered sacrificial formwork, or mould, is used to accommodate both structure and services within embedded conduits. This creates a complex lattice network which is made from a, primarily tensile, structural skeleton at the core surrounded by a secondary layer to allow for building services and compression foam insulation. This lattice network is embedded in a translucent outer skin. These two inner and outer skins laminate to one another periodically and are connected through flange geometries to create sufficient structural integrity in the skin at the macro scale (Fig. 5 right).
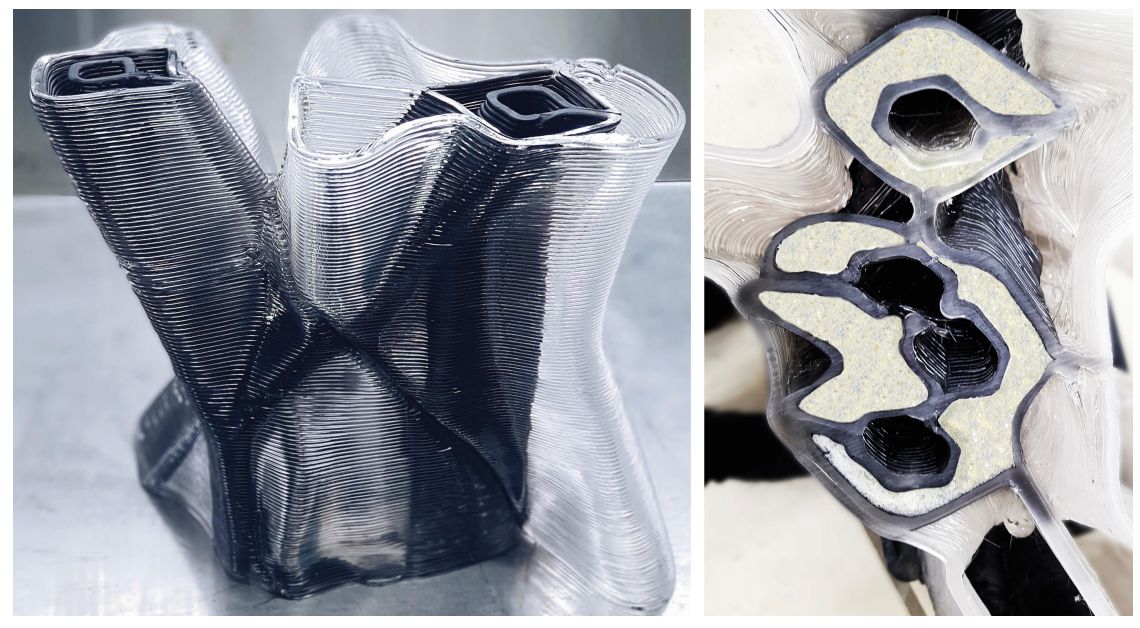

Fig. 5. 3F printed sacrificial formwork (left), preparation for carbon infusion (right)

Within the inner printed conduit, continuous carbon fibre elements are positioned and infused with an epoxy resin bonding the carbon fibre, steel connections and polymer sacrificial mould (Fig. 6).
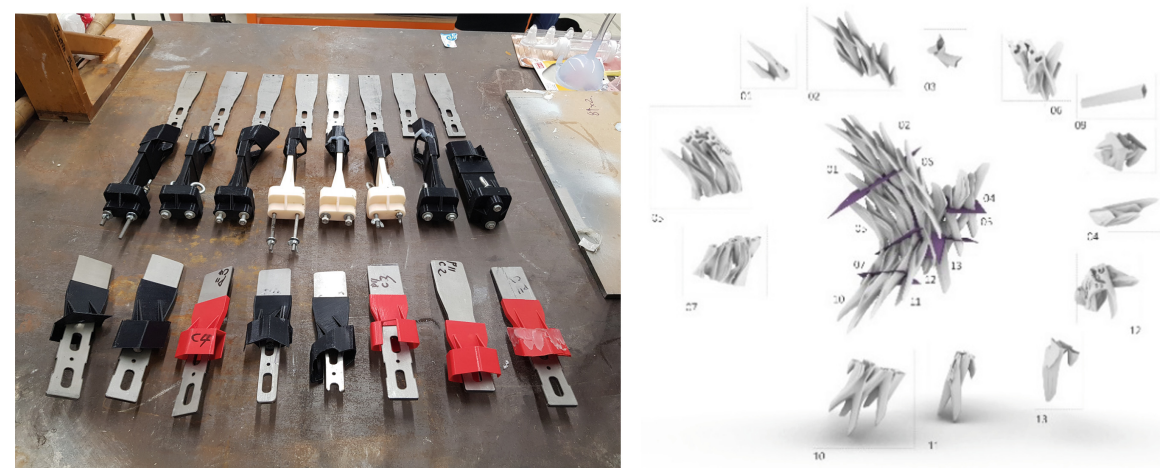

Fig. 6. Customised jigs for steel connections (left), assembly division \& joints (right) 


\subsection{BAAM Techniques and Geometric Implications}

Advancement in BAAM processes at the RMIT robotics lab in recent years has enabled the realisation of large scale algorithmically designed architectural prototypes such as Cloud Affects. Some of these technical enhancements, like improved start-stop printing and non-parallel/non-horizontal layer printing, are necessary to achieve substantial topological freedom of geometry and extreme cantilevering capacity. These improvements have also posed new challenges to the $3 \mathrm{D}$ printing process.

\subsubsection{D Printing Non-continuous Surfaces}

Robotic polymer pellet extruding enables relatively fast 3D printing through a high material flow rate. However, compared to filament printing, there is no material retraction in this process when a tool path stops. This results in the dragging of excess material between the endpoint of a tool path and the subsequent start point, creating polymer strings within the printed part. These strings cause accumulated defects between layers, affect the surface quality and results in possible print failure in large prints. Despite challenges created by this basic issue, start-stops are necessary for the fabrication of non-continuous surfaces, in particular surfaces with topological complexity. Through a design and fabrication feedback loop, this research introduced a new technique to resolve this by using flange connections between the independent layers of structure, services and skin conduits. This technique minimises the strings within the geometry by minimising start-stops and maximising continuous toolpath length, which results in enhanced print quality. As well as surface quality improvement, these flange connections increase structural connections between the conduits and transfer the loads more evenly to the fibre structure located in the core conduit.

\subsubsection{Directionality, Geometric Freedom and Extreme Cantilevering}

Conventional FDM printing where each layer is deposited horizontally, and perpendicular to the vertical axis of the tool, poses significant limitations to geometrical directionality. Small layer width to height ratios in large-scale polymer printing is essential to keep the weight of the 3D printed components down. However, this reduces the capacity to print cantilevering geometries with large draft angles. Non-parallel printing leverages the 6 axes of the robot to deposit non-horizontal layers in order to gradually change the layer heights and print plane orientation within the printing process. This enables the fabrication of geometries with more complex surface directionality and higher draft angles. This technique accompanied by temporary printed support material enabled significant cantilevers in the Cloud Affects project (Fig. 7).

These advancements require high accuracy and calibration for both hardware and software tools. Over the past 6 years of research and development, the RMIT robotics lab, in collaboration with industry partners, has developed several generations of polymer extruders with high accuracy and large deposition capacity. The latest generation of these extruders implements new functions and technical capacities such as a new shut-off valve system to reduce polymer strings as well as multiple heating and cooling zones in the extrusion barrel for controlled melting of the polymer. These hardware improvements have been in parallel with the development of software to better control and diagnose all 

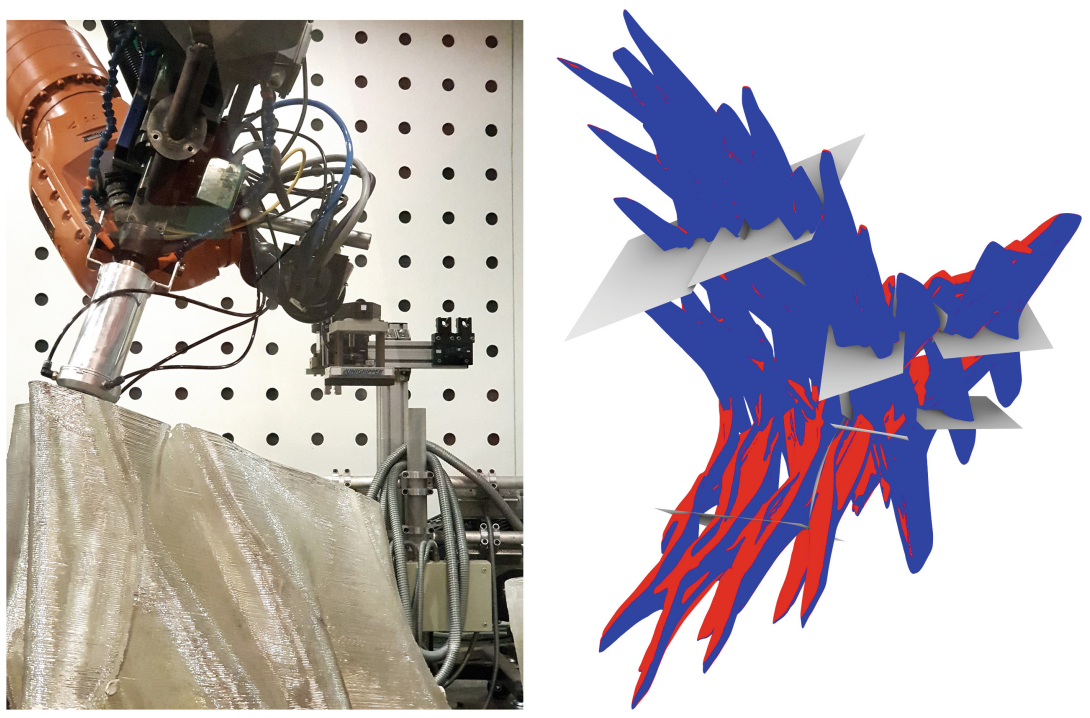

Fig. 7. Non-parallel printing (left), draft angle analysis (right)

aspects of the printing process, across multiple robotic platforms, materials and extruder types.

\subsection{Structural Design Implications}

The posited super composite of 3D printed polymer with infused carbon fibre structural members creates an integral link between the form, articulation and structure of the project. This requires both the geometry of the agentBody and its computational behavior to be designed through structural heuristics and their interaction with FEA analysis (Snooks 2020). This process establishes an inherently structural lattice which is subsequently fine-tuned and sized through an FEA analysis approach. This process evolved through the two projects to increase the accuracy of the analysis and efficiency of the structure.
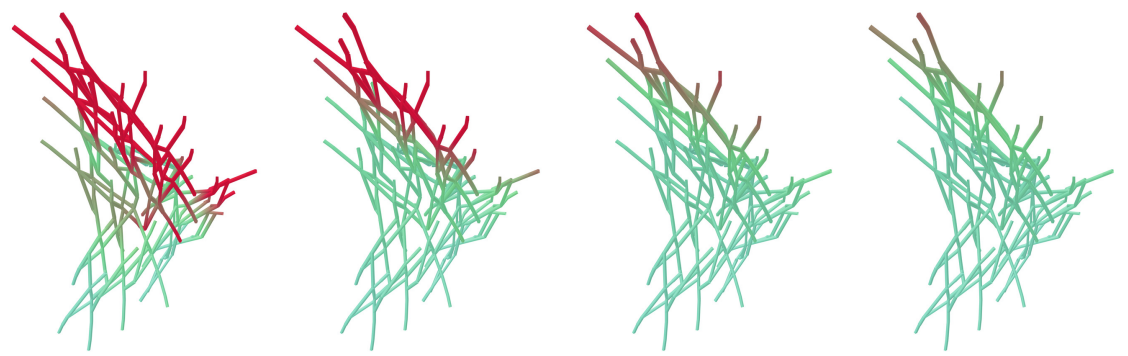

Fig. 8. Real-time structural feedback for fine-tuning of the amount and position of carbon fibre (front view) 
The structural setup of the two projects consists of an irregular cantilevering carbon fibre structure with prefabricated steel connections. Our approach to Cloud Affects was to simulate the stresses in the structural members. The overall shape of the structure is analysed as a truss successively using the FEA tools Millipede and Abaqus. We examined the structural strength of the assembly at the early form-finding stages using the Millipede in Rhino/Grasshopper platform. Global deformations and bending moments of the structure have been calculated and simulated for real-time fine-tuning of the carbon fibre sizing during the schematic design phase. This analysis took the overall weight of the structure, material properties of the resin and fibre and connection locations into account to calculate values of deformation at the extremes of the structure, where there is significant cantilevering. These simulations also determined the variable bending moments throughout the structure, the higher zones of stress indicated in red in Fig. 8, which enabled the distribution of variable section sizes of the carbon fibre elements. Later, we collaborated with the RMIT Center for Innovative Structures and Materials to calculate the structure in the phase of design development using the engineering FEA tool Abaqus. As opposed to the bending simulation in Grasshopper Millipede, the explicitdynamic analysis in FEA engineering software, Abaqus provided more accurate results for the design development. It offered the simulation and analysis of the bending process and values of maximum magnitude, mean stress and displacement by including different parameters such as overall structure weight, proportions of carbon fibre to resin, steel properties and other related boundary conditions. The gradient colour legend indicates the range of values for structural analysis result (Fig. 9). The accurate data helped us refine the design to ensure the appropriate safety and redundancy.
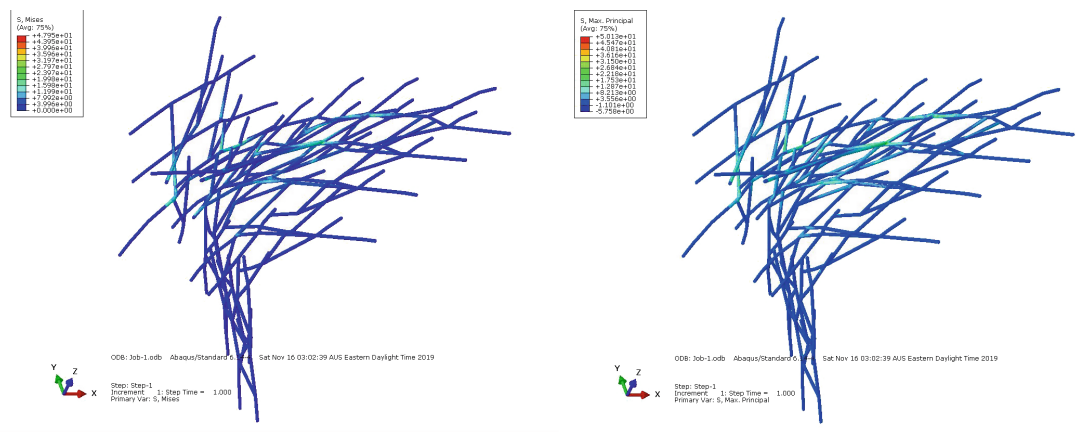

Fig. 9. Accurate simulation \& in-depth analysis of carbon fibre structure (rear view)

Although this analysis enabled us to design the overall structure by ballparking the deformation amount and weak moments of the structure, this method considers the carbon fibre as the only structural element, deliberately ignoring the strength of the $3 \mathrm{D}$ printed geometry to simplify the FEA approach. It, therefore, overestimated the required structural strength of the carbon fibre composite, resulting in oversized structural conduits, carbon fibre cross-section, resin volume and steel connections. Consequently, this process has been refined in the Unclear Cloud project for which we have developed a hybrid analysis that includes both the carbon fibre network and the compressive capacity 
of the polymer skin. This improvement in the simulation of the structure is helping us to create an accurate estimation of the required structural strength to reduce the amount of polymer, resin and fibre needed for the project.

The carbon fibre elements infused within the 3D printed conduits are continuous while the network analysis is undertaken as discrete segments of the structural lattice. Consequently, the discrete segments cannot be optimally sized without consideration of the continuous extent of the carbon element. This has led to the development of an approach, which we describe as fibre mapping, that optimises the layout of the continuous carbon fibre elements. This approach maps, or navigates, the fibres through the network relative to the specific bending moments of the discrete segments. This was initially developed as a manual iterative process in the Cloud Affects, before being encoded into an algorithmic approach in the Unclear Cloud project (Fig. 10).
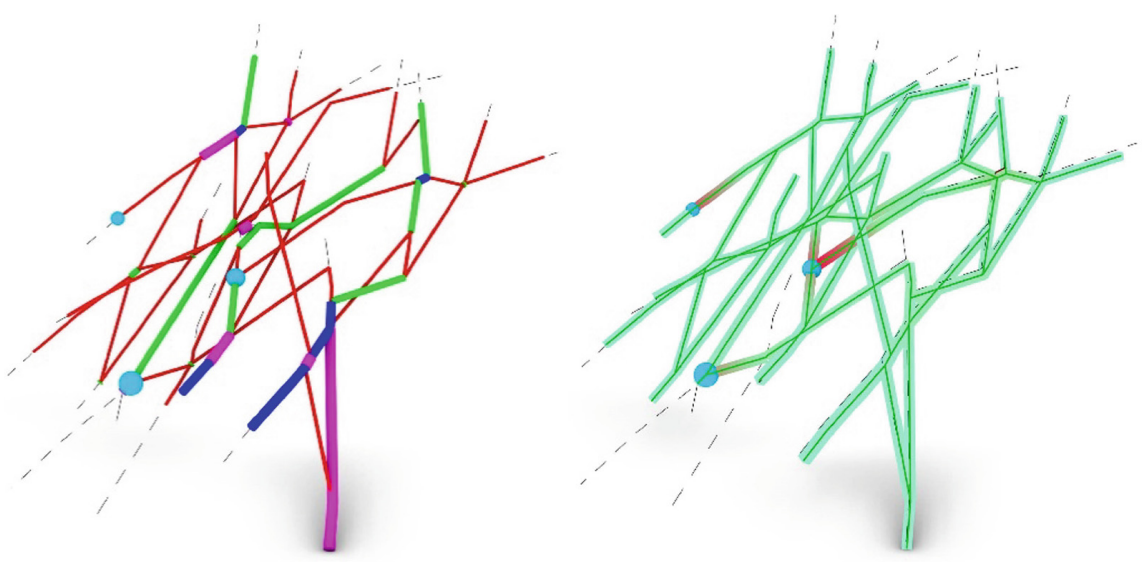

Fig. 10. Fibre mapping (right), improved bending moments (left)

\subsection{Jointing}

Logistics and polymer printing size limitations of the two projects require prefabricating parts which are assembled. Connecting these individual parts creates a substantial challenge and need for robust joints that create a continuity of structure equal to the strength of the carbon fibre elements. Other polymer printing issues such as shrinkage and warpage increase these challenges by creating uneven joints between panels. One approach in dealing with this issue is to overlap and conceal the seam lines with a built-in tolerance to connect individual panels side by side, horizontally. This method was used in a number of previous projects, including SensiLab, and B515 Studios (Snooks et al. 2020). The issue with this approach is the lack of structural continuity between panels. Another previous project, Floe (2018), used a continuous structural network fabricated from folded steel plates and a shingle-style arrangement of 3D printed polymer panels overlapping each other and connecting only to the steel sub-structure - not to one another. This method overcomes the issue of warpage and shrinkage by overlapping the 
panels at the edges where maximum warpage and shrinkage occurs during the printing process. However, the steel structure and mechanical connections are exposed, creating a disruption to the fluidity of the form and establishing discrete structure and skin. Cloud Affects overcomes this by using concealed steel fixing plates that create structural continuity across panel joints. In this method the integrity of the structure is preserved by creating a physical bond between infused carbon fibre and steel, the steel connections are concealed within the structural conduits and are connected to each other mechanically with minimum disruption to the skin surface. A mechanical fixing method was required for this project as it needed to be assembled and disassembled at multiple sites (Fig. 11).
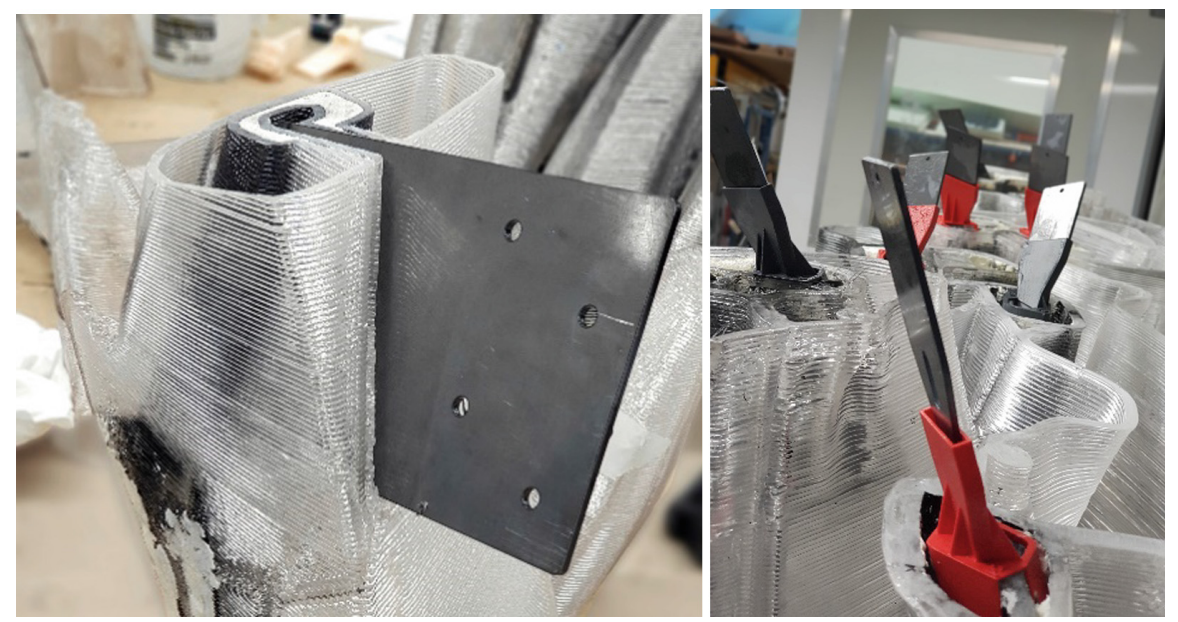

Fig. 11. Steel plate wall connection (left), implanted steel connections (right)

\section{Conclusion}

This research has outlined an approach to integrating carbon fibre structure within 3D printed polymer skins. This novel super composite strategy, of infusing carbon fibre within sacrificial formwork conduits, has evolved through a lineage of research projects at the Snooks Research Lab and contributes to a larger community of architects exploring robotic approaches to fabricating carbon fibre structures. To advance this research, we have developed innovative techniques and approaches in large-scale 3D printing, detailing necessary to infuse carbon fibre, and advances in digital tools to fabricate the necessary geometric complexity. The future direction of this work is intended to extend the research beyond proto-architectural demonstrator projects and explore the feasibility of this approach within the design and construction of larger-scale architectural projects.

\section{References}

Doerstelmann, M., Knippers, J., Koslowski, V., Menges, A., Prado, M., Schieber, G., Vasey, L.: ICD/ITKE research pavilion 2014-15: fibre placement on a pneumatic body based on a water spider web. In: Architectural Design, 5th edn. vol. 83, pp. 60-65. Wiley, New York (2015) 
Kwon, H., Eichenhofer, M., Kyttas, T., Dillenburger, B.: Digital composites: robotic 3D printing of continuous carbon fibre-reinforced plastics for functionally-graded building components. In: Willmann, J., Block, P., Hutter, M., Byrne, K., Schork, T. (eds.) Robotic Fabrication in Architecture, Art and Design 2018, ROBARCH 2018. Springer, Cham (2019)

Lynn, G.: Animate Form, pp. 40-41. Princeton Architectural Press, New York (1999)

Lynn, G.: Composites, Surfaces, and Software, Yale School of Architecture, New Haven, pp. 21-22 (2011a)

Lynn, G.: Chemical Architecture. DIALOG, pp. 27-29 (2011b). www.jstor.org/stable/41765685. Accessed 27 May 2020

Lynn, G., Friedman, T.: Intricacy. Intricacy: A Project by Greg Lynn FORM, pp. 27-29. Institute of Contemporary Art, Philadelphia (2003)

Miller, S.: Critical Mass: A Studio Tour with Greg Lynn. Composites and Architecture, DIALOG (2016). http://compositesandarchitecture.com/?p=4296. Accessed 25 May 2020

Snooks, R., Harper, L. (eds.): Printed assemblages. In: Burry, J., Sabin, J., Sheil, B., Skavara, M. Making Resilient Architecture, Fabricate 2020, pp. 202-209. UCL Press, London (2020)

Snooks, R.: Sacrificial formation. In: Wit, A., Daas, M. (eds.) Towards a Robotic Architecture, 5 th edn. Novato, California (2018)

Snooks, R.: Behavioral Formation: Volatile Design Processes and the Emergence of a Strange Specificity. ACTAR, New York (2020)

Open Access This chapter is licensed under the terms of the Creative Commons Attribution 4.0 International License (http://creativecommons.org/licenses/by/4.0/), which permits use, sharing, adaptation, distribution and reproduction in any medium or format, as long as you give appropriate credit to the original author(s) and the source, provide a link to the Creative Commons license and indicate if changes were made.

The images or other third party material in this chapter are included in the chapter's Creative Commons license, unless indicated otherwise in a credit line to the material. If material is not included in the chapter's Creative Commons license and your intended use is not permitted by statutory regulation or exceeds the permitted use, you will need to obtain permission directly from the copyright holder.

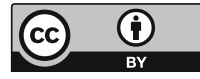

